Farum

Sociológico

\section{Forum Sociológico}

Série II

$27 \mid 2015$

Mobilidade científica \& imigração qualificada

\title{
Migración cualificada y políticas públicas en América del Sur : el programa PROMETEO como estudio de caso
}

MIGRACIÓN CUALIFICADA Y POLÍTICAS PÚBLICAS EN AMÉRICADEL SUR : EL PROGRAMA PROMETEO COMO ESTUDIO DE CASO ${ }^{1}$

Claudia Pedone e Yolanda Alfaro

\section{OpenEdition}

Journals

\section{Edição electrónica}

URL: https://journals.openedition.org/sociologico/1326

DOI: 10.4000/sociologico.1326

ISSN: 2182-7427

\section{Editora}

CICS.NOVA - Centro Interdisciplinar de Ciências Sociais da Universidade Nova de Lisboa

\section{Edição impressa}

Data de publição: 21 dezembro 2015

Paginação: 31-42

ISSN: 0872-8380

\section{Refêrencia eletrónica}

Claudia Pedone e Yolanda Alfaro, «Migración cualificada y políticas públicas en América del Sur : el programa PROMETEO como estudio de caso», Forum Sociológico [Online], 27 | 2015, posto online no dia 31 maio 2016, consultado o 29 março 2022. URL: http://journals.openedition.org/sociologico/ 1326 ; DOI: https://doi.org/10.4000/sociologico.1326 


\title{
MIGRACIÓN CUALIFICADA Y POLÍTICAS PÚBLICAS EN AMÉRICA DEL SUR: EL PROGRAMA PROMETEO COMO ESTUDIO DE CASO ${ }^{1}$
}

\author{
Claudia Pedone \\ - Universidad de Buenos Aires, Facultad de Filosofía y Letras, Instituto Interdisciplinario de Estudios de Género - CONICET \\ Yolanda Alfaro \\ Universidad Autónoma de Zacatecas, Unidad Académica de Estudios del Desarrollo
}

\begin{abstract}
Resumo
Diante das atuais transformações políticas, econômicas e sociais das migrações na Europa e na América Latina, o Equador se tornou um destino para profissionais altamente qualificados. No entanto, tanto no debate acadêmico quanto no político o discurso sobre esta migração qualificada aparece a reboque dos enfoques construídos para estudar as migrações de profissionais Sul-Norte com o uso de categorias analíticas, tais como, "fuga de cérebros" e "circulação de talentos" que se abstraem do contexto político-econômico no qual surge esse fluxo migratório. A partir do estudo do Programa "Prometeo" no Equador, analisamos diferentes perfis migratórios no âmbito dessa política pública e mostramos que a heterogeneidade que apresenta a denominada migração qualificada, através das variáveis de gênero, idade, origem regional e nacionalidade, nos permite refletir e debater sobre a relevância de abordar a análise da migração qualificada tendo em conta essas variáveis, a fim de construir outras categorias de análise.
\end{abstract}

Palavras-chave: migração qualificada, América Latina, crise europeia, perfis de migratórios

\begin{abstract}
Based on the current political, economic and social transformations of migration in Europe and Latin America, Ecuador has evolved into a destination for highly qualified professionals. However, both the academic debate and the political discourse on this skilled migration build upon constructed approaches to study the South-North migration of professionals with analytical categories such as "brain drain" and "brain circulation" that neglect the political-economic context in which the migratory flow arises. From the study of the "Prometeo" Programme in Ecuador, we set different migration profiles stablished by this public policy and show that heterogeneity presented by the so-called skilled migration -through the analysis of variables of gender, age, regional origin and nationality- allows us to reflect and discuss the relevance of addressing the skilled migration analysis taking into account other variables for the construction of other categories.
\end{abstract}

Keywords: skilled migration, Latin America, European crisis, migration profiles

\section{Resumen}

Frente a las transformaciones políticas, económicas y sociales actuales de las migraciones en Europa y América Latina, Ecuador se ha transformado en destino de profesionales altamente cualificados. No obstante, tanto en el debate académico como político el discurso sobre esta migración cualificada aparece en el marco de enfoques construidos para estudiar las migraciones de profesionales Sur-Norte con categorías de análisis como la "fuga de cerebros" y la "circulación de talentos" que se abstraen del contexto político-económico en el que surge dicho flujo migratorio. A partir del estudio del Programa "Prometeo" en Ecuador, damos cuenta de diferentes perfiles migratorios captados por esta política pública y mostramos que la heterogeneidad que presenta la denominada migración cualificada, mediante el análisis de las variables de género, edad, procedencia regional y nacionalidad, nos permite reflexionar y debatir sobre la pertinencia de encarar el análisis de la migración cualificada teniendo en cuenta estas variables en vistas a construir otras categorías de análisis.

Palabras clave: migración cualificada, América Latina, crisis europea, perfiles migratorios 


\section{Introducción}

El endurecimiento de los controles migratorios en EE. UU. y Europa y la profundización de la crisis económica global han impulsado ciertas modificaciones en la composición y dirección de las corrientes migratorias internacionales. En lo referente a las migraciones en América Latina, en los últimos años se registra una disminución de las salidas hacia Europa, el retorno de migrantes ecuatorianos, colombianos, bolivianos y argentinos que residían en España y la reconfiguración de los movimientos regionales de población.

Frente a las transformaciones políticas, económicas, sociales y territoriales actuales en el sistema migratorio transatlántico entre Europa y América Latina, países como Argentina, Brasil, México, Chile y Ecuador se han transformado en destinos de nuevas corrientes migratorias, configurando así una nueva cartografía de las migraciones cualificadas que demanda preguntarse, por ejemplo, ¿Cuáles son las causas de las migraciones de científicos y de profesionales desde los países del Norte hacia los países del Sur? ¿Cuáles son los países de destino de la migración cualificada Norte-Sur? ¿Cuál es el papel de los migrantes cualificados en las dinámicas Sur-Sur? y ¿En qué medida las políticas públicas que propician la movilidad de académicos y científicos altamente cualificados interviene en la reconfiguración de los flujos migratorios Sur-Sur?.

El desafío para responder a estas preguntas demanda la construcción de nuevas categorías de análisis. De acuerdo con los planteamientos de Bela Feldman-Bianco et al. (2011), la necesidad de construir nuevas categorías radica en reconocer que los significados y representaciones de las migraciones y los y las migrantes cambian en circunstancias históricas específicas.

Es por ello que en este trabajo pretendemos, mediante el estudio de las políticas y programas de atracción de migración cualificada en Ecuador, poner en debate y reflexionar sobre la pertinencia actual de las categorías de "fuga de cerebros", "migración cualificada" y "diásporas científicas", categorías que dieron origen a la producción científica en la década de 1960, para analizar la migración altamente cualificada en dirección Sur-Norte.

Nuestro estudio se llevó a cabo entre abril y octubre de 2014 y se priorizó el uso de estrategias metodológicas cualitativas. Se realizaron 38 entrevistas en profundidad y un cuestionario a toda la base de investigadores/as "Prometeos" como una herramienta de información complementaria. El cuestionario se envió a 504 investigadores/as beneficiarios/as el programa y lo respondieron 262 investigadores/as que equivale al $52 \%$ del total de la muestra, porcentaje que permite establecer una representatividad en las variables consideradas. Las entrevistas en profundidad tuvieron como criterios de selección: sexo, edad, nacionalidad, país de aplicación, área de conocimiento y la distribución territorial en diversas ciudades del país, tanto en Universidades como en Instituciones Públicas de Gobiernos Provinciales y Estatales.

En este artículo, en primer lugar, presentamos resumidamente el debate sobre las categorías que analizaron, desde la década de 1960, la migración cualificada y proponemos algunos elementos para construir nuevas categorías de análisis desde el enfoque de la interseccionalidad. En segundo lugar, hacemos una breve reseña del programa "Prometeo" como política pública del Gobierno del Ecuador orientada a la educación superior y a la investigación científica. Por último, exponemos algunos resultados de nuestro trabajo de campo que nos permite identificar y caracterizar los perfiles migratorios asociados a las movilidades de científicos/as teniendo en cuenta variables como: sexo, edad, procedencia, área de conocimiento e inserción laboral en origen y destino.

\section{Las categorías de análisis de la migración cualificada}

La aplicación del concepto de "fuga de cerebros" (Brain Drain) como categoría de análisis data de la década de 1960. En América Latina, los trabajos de Chaparro y Arias (1970), Rodríguez (1986), Oteiza (1996) y Pellegrino (2003) fueron claves para desarrollar este marco analítico, bajo el supuesto de que la migración internacional de personas con altas cualificaciones resultaba perjudicial para el desarrollo (crecimiento económico) de los países de la región. En la década de 1970, a partir de los estudios de la Comisión Económica para América Latina (CEPAL), los trabajos se centraron en analizar la migración cualificada en relación a los problemas que genera los modelos de desarrollo en la región en pos de demostrar que la pérdida de recursos humanos cualificados era una faceta más de un proceso de dependencia de los países del Sur respecto a los países capitalistas del Norte. En esta época los estudios sobre migración cualificada se centraban en las estrategias de los estados y el tipo de políticas necesarias para controlar la salida de mano de obra entrenada o limitar sus efectos negativos.

A partir de la década de 1990, el enfoque del estudio de la emigración de recursos humanos altamente cualificados tomó un nuevo rumbo, pues a partir de la implementación de las reformas estructurales que impulsaron el Banco Mundial (BM) y el Fondo Monetario Internacional (FMI) y el protagonismo de los organismos internacionales se empezó a delinear el enfoque de la "ganancia de cerebros" (Brain Gain) y la "circulación de talentos" (Brain Circulation), ambas categorías asociadas a la idea de que los migrantes altamente cualificados 
contribuyen de manera positiva en el desarrollo de los países de origen y destino.

En la última década las redes de profesionales altamente cualificados fueron abordadas bajo la categoría de "diásporas científicas". Esta categoría fue impulsada por la Comisión Europea y el Foro Mundial sobre Migración y Desarrollo en el marco del flujo Sur-Norte pero frente a la reciente reconfiguración migratoria en un contexto de crisis económica fue adoptada por algunos académicos y policy makers de los países de origen para resaltar el potencial de los inmigrantes cualificados en la transferencia de conocimiento científico a su país de origen.

Sobre todo ha sido aplicada para analizar aquellos programas de migración circular, gestionados como una herramienta de la política migratoria que promueve el enfoque en el que "todos ganan" ("win-win-win"); en el que los países de destino, los países de origen y los propios trabajadores migrantes puedan promover el desarrollo en los países de origen a través de la circulación de talentos, el retorno de las competencias, la creación de empresas, pero además, intenta reducir al mínimo la migración irregular, por lo que se ha convertido en una herramienta conveniente para los intereses de los países de origen en línea con las políticas económicas que promueven.

Las redes de profesionales altamente cualificados fueron abordadas bajo la categoría de "diásporas científicas". Esta categoría también fue propuesta por la Comisión Europea y el Foro Mundial sobre Migración y Desarrollo en el marco del flujo Sur-Norte pero frente a la reciente reconfiguración migratoria en un contexto de crisis económica fue adoptada por algunos académicos y policy makers de los países de origen para resaltar el potencial de los inmigrantes cualificados en la transferencia de conocimiento científico a su país de origen. No obstante, en la última década, tanto la circulación de talentos como las diásporas científicas han sido cuestionadas porque los supuestos beneficios en el desarrollo económico y político de los países no encuentran suficiente respaldo empírico (información), sino que por el contrario han sido utilizadas de manera acrítica, sin denotar la asimetrías que estructura la movilidad internacional de la élite profesional puesto que están insertas en la desregulación, la mercantilización y la nueva división internacional del trabajo (Delgado-Wise, 2014).

Aunque en los últimos años se han realizado esfuerzos académicos para desarrollar estudios sobre la movilidad de profesionales y científicos que aborden aspectos estructurales de la movilidad con aspectos de la micro-sociología del movimiento, estos han privilegiado la descripción de la subjetividad móvil sobre las causas y los efectos de este tipo de movilidad; es decir, sin establecer un análisis de la movilidad de profesionales y científicos altamente calificados hacia el norte global en el contexto de la globalización contemporánea y el capitalismo cognitivo (Sheller, 2011; Elliott y Urry, 2010).

Además, cabe señalar que el estudio de la movilidad en su sentido más amplio, no esboza la comprensión general del ejercicio del poder y todos los esfuerzos para orientar y dirigir cualquier aspecto que conlleva cómo el movimiento de la población en lugar del territorio se convirtió gradualmente en el centro del pensamiento moderno y la intervención gubernamental ${ }^{2}$. Así, aunque la crítica sobre las relaciones de poder intenta estar en el centro del campo de estudio, su planteamiento no se posiciona de manera crítica con la gubernamentalidad global, los estudios de la regulación o con las estructuras jurídicas pertenecientes a diversos tipos de movilidad, por lo que se reduce a una serie de consideraciones y herramientas conceptuales generales para el análisis de la descripción global de la movilidad.

De acuerdo con Mezzadra et al. (2014), el potencial del enfoque de la movilidad para estudiar la migración de profesionales altamente cualificados no está centrado en las preocupaciones crecientes sobre la velocidad de movimiento, sino en cómo en el contexto de la globalización contemporánea se ha establecido una re-organización espacial del trabajo. Esto significa que el estudio de la movilidad de profesionales altamente cualificados no puede limitarse a la descripción de los patrones de movilidad sino que debe abrirse a analizar los intereses políticos que están de por medio en la regulación de su movilidad; es decir, vislumbrar si se trata de un movimiento generado de manera voluntaria o forzada, si es temporal o a largo plazo, si es factible o problemático, etc.

Hay que resaltar que la migración cualificada tiende a ser discutida en términos generales y que muy a menudo pasa por alto la gran diversidad empírica de los casos y las muy diferentes situaciones de la migración calificada en dirección Sur-Norte y/o Sur-Sur. No es frecuente que al abordar esta temática se tenga en cuenta que, primero, entre estos dos flujos existen diferencias sustanciales, y, segundo, que detrás de la migración cualificada existen diversidad de perfiles de profesionales. Es oportuno resaltar que en un intento de complejizar el estudio de la reconfiguración de las migraciones en el sistema transatlántico, Cristina Vega et al. (2014) proponen el concepto de circularidad migratoria para abordar las migraciones de profesionales españoles al Ecuador en la actualidad y demuestran que el desplazamiento se debe más a la búsqueda de opciones laborales que al interés de construir redes de intercambio científico, ideas que se promueven desde las investigaciones que utilizan la categoría de "circulación de talentos".

Como se advirtió en la introducción, nuestro interés es: a) analizar la migración de profesionales 
altamente cualificados como una forma de movilidad específica dentro el marco de la migración Norte-Sur y/o Sur-Sur; b) considerar las particularidades y los significados que se le asigna a este tipo de movimiento por parte de los estados; y c) identificar las políticas que se construyen en torno a este tipo de movimiento y las representaciones que se generan de los profesionales altamente cualificados.

En tal sentido, consideramos que la perspectiva de la interseccionalidad tiene un potencial explicativo mayor que el enfoque de la movilidad en tanto abre el análisis al complejo entramado de diferencias que se tejen al considerar las dimensiones de raza/ /etnia/nacionalidad, clase y género a nivel macro, meso y micro. De esta manera la interseccionalidad nos obliga a centrarnos en articulaciones múltiples con el fin de encontrar puntos de convergencia en un tiempo y espacio determinado, es por ello que en lugar de reflexionar los niveles de análisis por separado se deben abordar en su articulación, de manera simultánea (Anthias, 2006; Ezquerra, 2008).

Si analizamos cómo la nacionalidad se cruza con el género y la clase ${ }^{3}$ en la organización de la migración de profesionales altamente calificados con dirección Norte-Sur y/o Sur-Sur podemos entender que las dinámicas de movilidad de profesionales adquieren connotaciones distintas. Siguiendo los postulados de Mohanty (2003) podríamos señalar que un profesional extranjero no se convierte en altamente cualificado única y exclusivamente porque alcanzó el cuarto nivel de formación académica, sino por las intersecciones que se construyen a partir de su condición de clase, lugar de origen, lugar de formación, campo de conocimiento y área de especialización.

La sola adquisición de cualificaciones no refleja las diferenciaciones sociales que operan de distintas maneras en las trayectorias de migración calificada (nivel micro), las relaciones sociales y de poder reproducidas en el contexto migratorio por las instituciones y las políticas (nivel meso); ni, mucho menos, las dinámicas de poder que atraviesa la generación de conocimiento en el capitalismo cognitivo (nivel macro). En otras palabras, el reconocimiento de la condición de migrante altamente calificado es resultado de una articulación de factores estructurales como de factores de reconocimiento y de pertenencia que tienen que ver con la política, la ideología, la práctica y la experiencia (Anthias, 2006).

En tal sentido, el panorama sobre las políticas de atracción de profesionales altamente cualificados se transforma radicalmente si aplicamos el enfoque interseccional, puesto que nos permite no sólo el análisis de la reestructuración de las dinámicas de la migración altamente cualificada en el contexto de la globalización, sino también cómo ésta es trazada y construida mediante mecanismos de control y de gestión (leyes, políticas e ideologías).
En tanto la perspectiva interseccional proporciona herramientas útiles para entender la complejidad y la diversidad de la migración altamente cualificada, nos hemos planteado centrar nuestro análisis en aspectos que revelan diferentes perfiles migratorios. Para dicho propósito hemos cruzado las cualificaciones de los profesionales con su nacionalidad, su condición de clase y género, para analizar: i) las diferencias de un grupo que globalmente se ha tratado como homogéneo, ii) las jerarquías y relaciones de poder que se construyen alrededor de las áreas de conocimientos y los lugares de procedencia de los mismos y, iii) entender algunos de los sesgos que caracterizar los debates que giran alrededor de la categoría de circulación de talentos.

Como veremos en las conclusiones, nuestro análisis se presenta como una propuesta de nuevos engranajes de interseccionalidad aplicados al análisis la migración altamente cualificada en pos de construir nuevas categorías de análisis.

\section{Ecuador como lugar de destino de migrantes extranjeros y retornados/as cualificados/as: el Programa Prometeo como política pública}

A partir de la promulgación de la Constitución del 2008, el gobierno de Ecuador comenzó a implementar las transformaciones a diferentes escalas y en diversas áreas de la producción, de la acción social y de los procesos institucionales que antes no existían.

Entre esas transformaciones, la educación se convierte en un objetivo central de las estrategias de desarrollo orientadas hacia la transformación estructural de la matriz productiva. De manera que el Plan Nacional de Desarrollo para el Buen Vivir 2013-2017 definió que la base del cambio de la matriz productiva, será la inversión en Investigación, Desarrollo e Innovación $(I+D+I)$, puesto que la generación de conocimiento, ciencia y tecnología constituirían el fundamento para superar las condiciones de dependencia que establece la actual estructura productiva al ser de tipo primario-exportadora (PNDBV, 2013-2017: 19).

La Secretaria Nacional de Educación Superior de Ciencia y Tecnología (SENESCYT), por su parte, definió políticas para impulsar la transformación de la matriz productiva, cuyo objetivo es incrementar las capacidades de investigación del país con equipamiento e infraestructura, institucionalidad y principalmente recursos humanos, por lo que se destinó el $0,66 \%$ del PIB en dos grandes campos de acción: i) investigación científica e innovación tecnológica y social y ii) formación de recursos humanos altamente calificados (SENECYT, 2012).

En relación al segundo campo se definieron planes programas y proyectos orientados a incorporar expertos de alto nivel a los institutos públicos de 
investigación, entidades públicas y sectores productivos priorizados. En este contexto, a partir del 2008 se puso en marcha el Programa Prometeo ${ }^{4}$ mediante becas en las siguientes áreas prioritarias: ciencias de la educación, ciencias de recursos naturales, ciencias de la producción-Innovación, ciencias de la vida y ciencias básicas. Estas apuntan a la transformación de la matriz productiva definidas por el estado ecuatoriano según el Plan Nacional del Buen Vivir.

Este proyecto se inicia a partir de un diagnóstico que identificó la necesidad de incrementar las capacidades de conocimiento de las universidades y escuelas politécnicas, institutos públicos de investigación, institutos técnicos y tecnológicos, entidades y organismos del sector público, en temas de investigación científica, docencia, innovación, desarrollo tecnológico y social en las áreas estratégicas del desarrollo nacional y regional, así como en los sectores productivos priorizados.

Es importante mencionar que en el Programa Prometeo se superponen políticas económicas, políticas de educación y políticas de I+D+I, por lo tanto, más que una acción política orientada a propiciar la atracción de profesionales altamente cualificados, se trata de una estrategia de desarrollo cuyos efectos generaron una inmigración temporal y de retorno de población altamente cualificada.

De acuerdo con los datos del Programa Prometeo, hasta diciembre de 2013, se vincularon a los Institutos Públicos de Investigación Superior, Universidades y Centros de investigación 573 Investigadores Prometeos; los cuales el $12,6 \%$ son ecuatorianos retornados, y el $87,4 \%$ restante está registrado en 44 nacionalidades distintas (España, Alemania, Reino Unido, Japón, Argentina, Bolivia, Colombia, Perú, India, Canadá, Francia, Portugal). Las nacionalidades más representativas son España con el $28,3 \%$; Venezuela con el $10,4 \%$ y Cuba con el $5,1 \%$. De acuerdo a los datos oficiales, para el 2014, la asignación de los proyectos de investigación estaba distribuidos de la siguiente manera: ciencias básicas 54\%, producción e innovación 20\%, recursos naturales $10 \%$, arte y cultura $8 \%$, ciencias de la vida $5 \%$ y ciencias de la educación $3 \%$.

\section{Perfiles migratorios \\ de los/as investigadores/as Prometeo vinculados a la reconfiguración de flujos migratorios}

Nuestro trabajo de campo con Investigadores/ /as Prometeo nos permitió identificar diversos perfiles migratorios que detrás de la categoría de "migración cualificada", presentan una alta heterogeneidad si se analizan desde una perspectiva interseccional, teniendo en cuenta variables como la edad, el género, la nacionalidad/procedencia regional y la pertenencia a clase social.
En cuanto a la variable género el Programa tiene una abrumadora presencia masculina, sólo el 32\% de las Prometeos son mujeres. Los resultados de nuestra muestra concuerdan con los datos generales del Programa, especificados anteriormente. Entre los 246 participantes en la investigación, el 71.5\% fueron hombres y el $28.5 \%$ mujeres. La procedencia regional la encabeza la Europa Mediterránea con una participación del 44\%, principalmente la llegada de profesionales de España y luego un perfil migratorio procedente de América Latina del 35\%, donde predominan los/as científicos/as de Venezuela, mientras que la población ecuatoriana cualificada retornada sólo llegaba a un $9 \%$.

Teniendo en cuenta estos datos de procedencia regional, predomina el uso del idioma castellano en un $89,4 \%$ y el $10,6 \%$ para el inglés. La edad media de la muestra es de 48 años en un rango entre 29 y 76 años. En cuanto a las áreas de investigación la muestra corrobora los porcentajes que se definen como estratégicas en el cambio de la matriz productiva: 34\% Ciencias de la Producción-Innovación, $21 \%$ Ciencias de Recursos Naturales, $20 \%$ Ciencias de la Vida, los menores porcentajes se encuentras en las Ciencias Sociales con $12 \%$, Arte y Cultura $6 \%$ y Ciencias de la Educación $3 \%$.

La primera aproximación de este análisis fue conocer los canales a partir por los cuales los/as profesionales habían conocido y tomado contacto con el Programa. Si bien, desde la Gerencia se enfatiza en que la difusión del Programa se hace por medio de las embajadas y los vínculos que brinda la cooperación internacional, nuestro trabajo de campo corroboró que estos mecanismos institucionales de difusión son los menos eficaces. Los canales de difusión más efectivos para el conocimiento y contacto con el Programa son las relaciones personales y los medios de comunicación y redes sociales. En cuanto a los vínculos previos con Ecuador, predominan los de tipo académicos y de amistad, aunque es importante señalar que el $44 \%$ de los/as profesionales de la muestra no tenían ningún vínculo ni conocimiento previo con Ecuador.

El conocimiento del Programa se da, principalmente, mediante la comunicación entre investigadores/as, que a su vez, están propiciando redes de profesionales calificados, que organizan las estancias en Ecuador procedentes de los mismos países e incluso de las mismas universidades y que tanto desde la Europa Mediterránea como desde algunos países de la región, como es el caso de Venezuela, responden a una estrategia de inserción laboral, debido a la crisis económica y financiera que sufren los sistemas educativos y de ciencia y tecnología en sus países de origen.

El programa lo conocí un día que mi directora de tesis vino a comer a nuestra 
casa y le comentaba que yo ahora estaba sin trabajo en España, y le decía que si no sabía de alguna cosa que podía hacer y entonces me comentó lo de Prometeo (Mujer, 45 años, nacionalidad española, aplicó desde España, Ciencias de la Vida).

Por mi mujer que es también Prometeo, ella se enteró. $Y$ bueno como a ella en principio le respondieron muy rápido, entonces apliqué yo, y decidimos venir para aquí (Varón, 53 años, nacionalidad argentina, aplicó desde España, Ciencias de la Producción e Innovación)

Por un lado me llegó una información por una página web que publicó la Universidad de Los Andes, en la ciudad de Mérida, hablaban en qué consistía el proyecto y ofreciendo a profesores de la universidad y más tarde un compañero de institución fue seleccionado, entonces ahí, él me dijo que porque no veía la posibilidad de también de postularme, y fue cuando decidí postularme y pasé la selección (Mujer, 45 años, nacionalidad venezolana, aplicó desde Venezuela, Ciencias de la Vida).

Por otro lado, existe un grupo de investigadores/as que llegan a Ecuador por vínculos académicos previos, sobre todo con profesionales ecuatorianos/as que han realizado posgrados y estancias de investigación en el exterior.

Como adelantáramos, a partir de esta caracterización general los resultados de nuestra investigación develaron que dentro de la denominada "migración cualificada" existen una variedad de perfiles y una heterogeneidad dentro de los mismos. A continuación describimos las características de los/as protagonistas de estos desplazamientos:

Perfil migratorio altamente cualificado procedente de América del Norte, India, Norte de Europa

Entre los y las científicas que componen este perfil la edad oscila, preferentemente, entre 55 y más de 70 años. Son profesionales con alta cualificación que a partir de sus años sabáticos o su jubilación, el Programa Prometeo les otorga la posibilidad de conocer parte de América Latina, consolidar vínculos académicos iniciados años atrás y aprovechar a poner en marcha proyectos de investigación atractivos para el proceso de transformación de la matriz productiva de Ecuador.

A nivel de género predominan los varones que, en su mayoría, llegan acompañados solamente por sus parejas. Este perfil de investigadores/as son quienes valoran más positivamente las iniciativas del Gobierno del Ecuador y minimizan los inconvenientes que suelen presentarse en la puesta en marcha de los proyectos de investigación debido a la falta de infraestructura y equipamiento o las trabas burocráticas en las instituciones de acogida.

\section{Me da envidia y más en este momento en} que veo la política, es que inevitablemente uno compara con lo que conoce más, veo la política de mi país; la política científica penosa, lamentable de mi país, me da mucha envidia, por eso estoy siempre tan dispuesto a colaborar en todo lo que me pidan. Ven a ser jurado, voy. Ven a la reunión de biodiversidad, a lo que haga falta. Por eso yo sería muy estricto en la selección, es decir, una persona no puede venir aquí a hacer curriculum científico, de ninguna manera, para eso te tienes que ir a Estados Unidos o a Europa, o Australia, o yo que sé, aquí hay que venir con un cierto bagaje, y con una cierta experiencia, y con la mochila y venir a vaciar la mochila, no a llenarla (Varón, 62 años, nacionalidad española, aplicó desde España, Ciencias de la Vida)

Es oportuno resaltar que no se reconocen como migrantes, enfatizan en jerarquizaciones y diferencias de clase social y asocian la categoría de inmigrante a personas pobres y de una precaria inserción laboral. Generalmente, estos/as científicos/as llegan a Ecuador por estancias cortas, desde 4 meses a 1 año. Este perfil podría adscribirse a la categoría que algunos autores trabajan bajo la perspectiva de movilidad académica internacional (Cresswell, 2010; Elliott y Urry, 2010; Sheller, 2011); no obstante, estos científicos aprovechan la posibilidades de esta política pública para poner en marcha sus proyectos de investigación con mayores recursos económicos y con salarios altamente remunerados. Cabe señalar que estas condiciones, a partir de la crisis socioeconómica del 2008, han mermado tanto en América del norte como en Europa.

Adscribirse a la perspectiva de movilidad académica internacional implicaría invisibilizar las relaciones de poder que subyacen bajo los desplazamientos de profesionales altamente cualificados y excluirlos del análisis de la actual reconfiguración de los flujos migratorios en América Latina.

Perfil migratorio producto de la crisis europea, específicamente, España e Italia

La llegada de científicos/as desde España e Italia presenta una heterogeneidad que nos ha permitido distinguir dos perfiles migratorios atravesados por la edad y las posibilidades de inserción laboral en los lugares de origen.

Estos/as profesionales tienen una edad oscila entre los 29 y 45 años y buscan en el Programa 
Prometeo una opción laboral frente a la crisis socioeconómica y a las escasas posibilidades de inserción académica en sus lugares de origen. Sus trayectorias de posgrado, son nacionales entre los/as más jóvenes y entre los que pasan los 40 , en la mayoría de los casos, han realizado estudios de doctorado y posdoctorales en otro país de Europa o Estados Unidos.

Su opción por Prometeo responde a la imposibilidad actual de acceder a becas y estancias en EE.UU y Europa, como hasta hace unos pocos años, y pretenden mantener el vínculo entre Ecuador-España como una estrategia de sobrevivencia, puesto que planean un retorno a medio o largo plazo.

Una vez que finalicé mi doctorado en Granada solicité el proyecto Prometeo y me contestaron inmediatamente y vine para acá. Yo tenía otras expectativas, de hacer una estancia postdoctoral en la Universidad de Suiza, muy interesante, pero consideré, que esto era mucho más interesante por el hecho de que te desplazas a un continente distinto. Yo soy una persona joven, y tenía ganas de conocer y más que nada, porque dices: Es la oportunidad de hacerlo ahora, si no, no puedes (Mujer, 29 años, nacionalidad española, aplicó desde España, Ciencias de la Vida).

Dentro de este perfil hay profesionales entre los 29 y 37 años que llegan solos con proyectos individuales y con casi nulas posibilidades de insertarse en las universidades españolas e italianas como docentes y/investigadores/as. Son muy críticos con la gestión del Programa en cuanto a retrasos en los pagos mensuales, a la entrega de informes mensuales, reembolsos de visitas académicas, debido a que pretenden que el Programa funcione como una beca posdoctoral.

Es seguro que como gestión es bastante extraña, respecto a las becas que estamos acostumbrados en otros lugares. Es un sistema de control demasiado estrecho. En ningún proyecto financiado, por ejemplo en mi experiencia en Italia, se pide un informe mensual. Esto es muy extraño porque sobre todo, en un proyecto de investigación científica básica no se puede prever qué va salir. A lo mejor nos puede salir mal, porque si no, no sería investigación. $Y$ pensar de planear, mensualmente un informe donde se tiene como requisito para ser pagado, de que se respeta la matriz de actividades, es algo muy problemático (Varón, 37 años, nacionalidad italiana, aplicó desde Italia, Ciencias Básicas).
Yo creo que hay demasiada burocracia, a mí... cuando estás trabajando a gusto y sobre todo cuando tienes mucho trabajo, sobre todo cuando tienes que hacerlo en seis meses; he perdido mucho tiempo con burocracia. Los informes mensuales son tremendos y además yo dudo mucho de que los puedan leer, creo que no los leen. Porque no tienen esa capacidad de leer tanto informe, entonces es mucho tiempo que dedicas, y mucho papel que gastas y yo dudo mucho de que tenga una utilidad (Mujer, 45 años, nacionalidad española, aplicó desde España, Ciencias de la Vida).

Otros con edad entre 38 y 50 han llegado en ocasiones con sus familias, toman la estancia de Prometeo como una posibilidad para cumplir con requisitos académicos en España sobre las estancias en el extranjero, con la ventaja comparativa de muy buen salario, prestaciones de servicios de salud y de residencia por los primeros seis meses. Por lo cual, paralelamente a cubrir las exigencias académicas en origen, su estancia como Prometeo les permite poner en marcha estrategias de ahorro, ya que la mayoría sigue cobrando sus salarios en España.

Algunos/as investigadores/as no han logrado reinsertarse en España luego de algunos posgrados en el extranjero, por lo cual, el Programa Prometeo se ha convertido en una estrategia laboral ante la imposibilidad de inserción en sus universidades de origen.

Estoy en mi tercer post doc. He hecho un post doc en Francia, hecho un pos doct en España y hecho un post doc en Irlanda, y en este momento, estaba estoy presentando a oposiciones en Francia, Suecia y Chile y dentro de todo el rango de oposiciones me presenté a Prometeo. En España nunca estuve fijo en ninguna universidad, nunca. Yo hice el doctorado en Irlanda, luego hice una pequeña estancia de un año en España, pero no conseguí financiación, luego hice dos años más de pos doc en Francia, luego conseguí una beca de la Junta de ampliación de estudios. Estuve tres años de pos doc en el Instituto de Tecnología Molecular, en Valencia, y luego conseguí un pequeño contrato de diez meses en otro departamento en la universidad de Dublín. De hecho, llevo dos años intentando conseguir la Ramón y Cajal, y aunque consigo muy buena puntuación, no es suficiente para entrar en la calificación. Soy demasiado viejo. Ya me pasé la edad (Varón, 45 años, nacionalidad española, aplicó desde España, Ciencias de la Vida). 
Los/as investigadores/as Prometeos que arriban con familia, en sus testimonios exponen las dificultades que han tenido para insertar a los y las hijas en el sistema educativo y encontrar vivienda. El propio Programa no contempla la noción de familia asociada a la llegadas de estos/as científicos/as, por lo cual no existe apoyo logístico en la instalación familiar.

Un niño yo creo que es muy complicado, y más difícil con un niño de nueve años que con un niño de cuatro o de tres. Yo creo que lo que más les ha costado, es que se han separado de sus amigos. Sus amistades, no sé. Yo creo que también a Clara, le supuso mucho el ir a una escuela donde no conocía a nadie, para ella fue como un abismo. Lo que más le costó fue eso, y la comida, le costó mucho. El tema de la alimentación fue duro.

(La decisión familiar) "fue lo más difícil. Yo tengo un hijo de 16 años. No quería venir por supuesto. Estaba terminando la escuela, con sus amigos. Lo convencimos diciendo que él en dos meses, que estudiaba ya tenía vacaciones y aprobaba todo el año. Lo mismo con mi esposa, nunca vamos solos. $Y$ entonces decidió venir, pero la condición es que si venía, tenía que tener trabajo aquí. Esa fue la condición que yo puse para mí como Prometeo, si no tampoco venía. Porque nueve meses era mucho tiempo de estar solo".

Es llamativo observar que son las mismas preocupaciones que las familias migrantes ecuatorianas tienen a la hora de reagrupar a sus hijos/as en los lugares de inmigración. No obstante, estas familias tienen en Ecuador asegurada la regularidad jurídica de toda la familia, y pueden organizar estancias más prolongadas con posibilidades de inserción laboral y sin problemas de irregularidad jurídica. La migración familiar dentro de las investigaciones sobre migración cualificada es un campo de estudio descuidado, la mayoría se centran en analizar estos desplazamientos como proyectos autónomos, donde la familia queda invisibilizada, en las políticas que los originan y en el impacto tanto en los lugares de origen como los de destino ${ }^{5}$.

Perfil migratorio producto de la reconfiguración de los desplazamientos de migración cualificada latinoamericana

Este perfil está estrechamente vinculado al retorno de los y las migrantes latinoamericanos que residían en Europa y Estados Unidos, en algunos casos, son retornos temporales y en otros definitivo, pero es una forma de buscar lugares de inserción cualificada ante el deterioro de las condiciones labo- rales en el ámbito científico tecnológico en Europa y Estados Unidos.

Estas estrategias migratorias son viables porque existen políticas en ciencia y tecnología de atracción en algunos países de la región latinoamericana, como es el caso de Ecuador, Argentina y Brasil, con políticas más progresistas y Colombia con una política de repatriación de profesionales con un marcado corte neoliberal ${ }^{6}$.

Este perfil latinoamericano es más variado en edades, procedencias y países de aplicación?. En este sentido, hay una característica en común que se refiere a que los/as científicos latinoamericanos/as han realizado sus carreras de posgrado en Estados Unidos, Canadá y Europa, y en este momento están ensayando diversas estrategias: a) como repatriados/as que buscan un retorno temporal y/o definitivo a su país; b) como retornados/as buscan inserción laboral en diversos países de la región y c) estrategias de inserción académica que incentivan las migraciones intrarregionales a lugares que se han convertido en nuevos destinos como Ecuador, Brasil, Chile, Argentina y México.

\section{Perfil migratorio de Investigadores/as Ecuatorianos/ as Retornados/as}

Nuestro estudio reveló que este perfil migratorio no sobrepasa el $10 \%$ de los/as profesionales procedentes de América Latina y mantiene el equilibrio en cuanto a edades y paridad de género. Son docentes e investigadores/as que han realizado sus trayectorias académicas doctorales y posdoctorales en exterior y que han combinado, en la mayoría de los casos, con trabajos precarios en los inicios de sus carreras en los lugares de emigración.

La mayoría se ha vinculado al Programa Prometeo como una estrategia de retorno, los más jóvenes proceden de ámbitos académicos europeos donde no han logrado una inserción fija y los de mayor edad ven la oportunidad de insertarse en origen como una forma de cumplir con el proyecto de retorno a largo plazo planeado en los inicios de su migración. En general, tienen con un alto compromiso político debido a los procesos de cambio que vive nuestra región $y$, en particular, Ecuador y este hecho ha sido un factor importante para definir el retorno temporal y/o definitivo.

Pienso que es interesante venir al Ecuador a radicarse por el momento, sobre todo por toda esta efervescencia que hay. Tenemos las condiciones para trabajar. Yo al menos aquí en la UTPL, he tenido todo a mi disposición, desde el punto de vista tecnológico, tengo las últimas versiones de los aparatos, que ni siquiera en los laboratorios de Francia hay. Entonces veo que todavía hay mucho que 
hacer, porque si bien en tecnología podemos acceder a las últimas versiones, pero en cambio, todavía falta mucho para instaurar una filosofía de investigación por formar a la gente como se debe para hacer formación de calidad. Entonces veo en eso un reto (Mujer, 52 años, nacionalidad ecuatoriana, aplicó desde Francia, Ciencias de la Vida)

Bueno, cuando decidimos volver al Ecuador con mi esposa, regresar después de haber sido profesores por trece años en Estados Unidos, pues empezamos a buscar alternativas de trabajo y justamente ahí nos hablaron del programa Prometeo. Sabíamos que en las humanidades no había mucho espacio, pero sabíamos también que había algunos miembros que intentaban generar un espacio para las humanidades. Yo creo que fue un momento, de suerte en el que se abrió justamente ese espacio, para las humanidades (Varón, 48 años, nacionalidad ecuatoriana, aplicó desde Estados Unidos, Humanidades)

Es oportuno resaltar que entre los/as científicos/ecuatorianos con una trayectoria académica prolongada en Europa y Estados Unidos son muy críticos/as con las desigualdades de cargas horarias y diferencial de salarios que origina la llegada de Prometeos frente a sus pares ecuatorianos en las instituciones de acogida y también con la calidad de los profesionales que están llegando y con los criterios de selectividad del Programa.

Yo he visto y de los Prometeos dependiendo también de quién sea, porque realmente no sé cómo se haya hecho la selección, pero veo que en muchos casos, no están calificados. Bueno, de lo que yo he visto que llegó conmigo, son pocos los que realmente los consideraría como investigadores interesantes que puedan aportar. El resto no lo es. Inclusive ha habido que formarles aquí. Entonces mi país está pagando para que haya gente que aporte y no lo contrario, no vamos a pagar para formar a otros. Entonces, bueno. Sí me he cuestionado como está funcionando lo de los investigadores cualificados"(Mujer, 52 años, nacionalidad ecuatoriana, aplicó desde Francia, Ciencias de la Vida).

Hay muchos profesionales doctores muy jóvenes, me parece que han venido muchos. Que quizá es su primera experiencia de investigación, después de su disertación, entonces claro, lo que ven ahí es algo que agregar al currículo. No quisiera generalizar, pero sí creo que hay varios Prometeos que están en esa línea. Ahora, realmente están involucrados en tratar de generar cosas y dejar cosas. Pero creo yo que el programa debería tratar de buscar otro tipo de perfiles, el perfil de investigadores, que tienen experiencia o que traen experiencia de otros lugares y tratar de reconectar esa experiencia al país. Y por ahí yo creo que hay un espacio en que se debería trabajar más. (Varón, 48 años, nacionalidad ecuatoriana, aplicó desde Estados Unidos, Humanidades)

Debido a la competencia generada a partir de la llegada de científicos altamente cualificados/as, los profesionales más jóvenes de este perfil encuentran obstáculos para insertarse laboralmente en sus áreas de especialización, lo cual conlleva replantearse su retorno definitivo y a ensayar nuevas estrategias migratorias como las becas de la SENESCYT para continuar estudios de posgrados en el exterior o plantearse la re-emigración. Este hecho nos lleva a señalar que el Programa no contribuye a la repatriación de recursos humanos.

\section{Perfil migratorio producto de la crisis sociopolítica de Venezuela}

Si bien la causa principal de la llegada de profesionales procedentes de Venezuela es el deterioro de las condiciones socioeconómicas en el país y en sus universidades, existen dos perfiles marcados preferentemente por la edad.

Un segmento de profesionales a partir de los 55 años jubilados y que la inserción en el Programa Prometeo les permite acceder a mejores condiciones de vida frente a pensiones devaluadas. Otro perfil que va desde los 30 años hasta los 50 con una trayectoria académica consolidada que optan por esta migración debido a los mayores salarios, para ello, han pedido permiso de un año remunerado o no remunerado en sus lugares de trabajo, sin embargo, muchos consideran renunciar si logran una inserción a mediano o largo plazo en Ecuador.

Ambos perfiles toman al Programa Prometeo como una opción laboral y de vida ante las condiciones socioeconómicas deterioradas y condiciones político-universitarias conflictivas en origen. Sus planes son de retorno a largo plazo y de un asentamiento a mediano plazo en Ecuador, por ello, tienen una mejor valoración de la gestión del Programa y ponen en marcha estrategias novedosas ante las dificultades académicas, institucionales y de infraestructura encontradas en las Instituciones de Acogida.

Para mí está descartado regresar a Venezuela, aunque no estoy seguro todavía de quedarnos aquí. No me desagrada. Nosotros hemos pasado por una etapa de descompre- 
sión, de salir de los rollos, de las paranoias, aprender a vivir una vida normal, otra vez aquí y la verdad eso lo he disfrutado mucho. Salir de noche sin que te atraquen, sin tener una pistola en la cabeza, estoy feliz (Varón, 54 años, nacionalidad colombiana, aplicó desde Venezuela, Ciencias de la Vida).

Mi esposo y yo somos profesores, y de hecho todavía tenemos nuestros cargos fijos en la Universidad Central de Venezuela y solicitamos un permiso no remunerado por un año. Hay una situación concreta nacional en Venezuela. Una de las cosas es que los sueldos para los profesores universitarios son muy bajos, y en realidad no alcanzan para mucho. Pero además estábamos viendo un deterioro progresivo, y eso nos daba mucho temor, además de eso, hay una situación de inseguridad grave en Venezuela (Mujer, 39 años, nacionalidad venezolana, aplicó desde Venezuela, Ciencias de la Vida).

El perfil de profesionales más joven llega a Ecuador con familia, hijos pequeños, y tienen las mismas demandas que el perfil de profesionales españoles, hablan de la deficiencia de la educación y la dificultad para encontrar viviendas adecuadas en las ciudades intermedias y pequeñas.

La adaptación fue más difícil de lo que yo me imaginé. Pensé que iba ser un poco más llevadero, pero nos ha costado porque, a mi hija le ha costado adaptarse a la guardería, y ya está mejor y como todo que requiere un tiempo, creemos que como aquí se habla español y somos muy parecidos. No. Somos, diferentes. Mi impresión es que los profesionales en la educación no son muy buenos. Esa es la impresión que me ha dado en general las guarderías y la experiencia, particularmente en esta, que supuestamente es una de las mejores, es que a mí me parece que no tienen una rutina. En Venezuela, hay mucho las rutinas, para que los bebés aprendan cosas. Pasan cosas y no te enteras. Yo siento que la saco y la meto en la caja negra (Mujer, 36 años, nacionalidad venezolana, aplicó desde Venezuela, Ciencias Básicas).

En general, a medida que logran renovar sus vinculaciones como Prometeo o la inserción laboral en la docencia e investigación el retorno a corto plazo se desdibuja, y algunos/as científicos/as han renunciado a sus cargos en Venezuela. Otra estrategia es buscar posibilidades en otros países de la región como por ejemplo Brasil.

\section{Algunas reflexiones finales}

El Programa Prometeo si bien no es una política pública asociada a la Política Migratoria Integral, sus repercusiones reflejan las transformaciones que tienen lugar en la reconfiguración de los flujos migratorios Norte-Sur y Sur-Sur. Los resultados de nuestro trabajo de campo revelan que este Programa al permitir la inserción laboral en el sistema científico-tecnológico y en la educación superior del Ecuador, se ha convertido en una salida laboral para migrantes cualificados/as europeos/as, latinoamericanos/as y ecuatorianos/as retornados/as afectados por la crisis socioeconómica en Europa.

Al igual que otros flujos migratorios la articulación y dinámica de las redes migratorias son un elemento clave para entender el proceso de atracción y/o inserción laboral de los/as profesionales altamente cualificados/as. Este hecho permite trascender la idea de que en la migración cualificada prevalecen los proyectos migratorios individuales, y refleja la dimensión social y familiar de estos procesos migratorios.

La circulación de la información entre las redes de científicos/as regionales y/o nacionales hace que, en algunas áreas del conocimiento, la llegada de investigadores/as responda más a la oferta de los postulantes que a la demanda del propio Programa. Esta situación ha desvirtuado el perfil académico que la política pública buscaba en sus inicios.

En relación al género, las relaciones asimétricas que se dan en el ámbito científico-tecnológico a nivel mundial, el esquema se repite en Ecuador, con una preeminencia financiera en las ciencias básicas y una hegemonía masculina. Además, es oportuno resaltar que las mujeres con cargas familiares llegan junto a sus parejas que también son Prometeos o como acompañantes de los varones. Las científicas que llegan con proyectos individuales son solteras o con hijos ya independizados y constituyen un porcentaje muy bajo de representatividad.

Teniendo en cuenta la pertenencia a clase social, en general, los y las investigadoras Prometeo no se auto-identifican con la categoría de inmigrante. En el perfil altamente cualificado, procedente de América del Norte y Europa del Norte, sus prácticas discursivas alegan que la temporalidad de su movilidad les impide considerarse como inmigrantes, puesto que en este discurso subyace un marcaje de clase que los conduce a asociar su llegada a Ecuador como una condición de pertenencia al "mundo de los científicos/as globales".

Un aspecto que nos permite matizar o rechazar la idea de que no se trata de un tipo de movilidad en el contexto de la globalización, sino de una dinámica migratoria se debe a que muchos de los científicos que fueron atraídos por el Programa Prometeo, terminaron planteándose un proyecto 
de vida a largo plazo en el país, al insertarse en la academia ecuatoriana con sus propias líneas de investigación.

Uno de los perfiles que refuerza estas jerarquizaciones es aquel que procede de la Europa Mediterránea, que a partir de narrativas, en ocasiones, con tintes neocoloniales, no reconocen que su arribo a Ecuador forma parte de un proyecto migratorio laboral. Entre estos/as profesionales prevalece el desconocimiento sobre el contexto político y económico del Ecuador que busca la política pública de la cual son beneficiarios.

Los/as investigadores/as procedentes de Venezuela son más explícitos a la hora de exponer sus intereses académicos y sus proyecciones de vida a mediano y largo plazo en el país. Si bien, la llegada de académicos/as desde Venezuela se ha visto facilitada por los vínculos construidos entre los dos gobiernos en la última década, en las entrevistas existía una reticencia a explayarse sobre las causas político-económicas en Venezuela que los/as condujeron a la migración a Ecuador, por temor a tener inconvenientes en la renovación de sus becas o en una futura inserción laboral en el país andino. Por ello, una variable en la cual debería profundizarse son las relaciones políticas entre los dos países sobre el tema migratorio.

Cabe destacar que la inserción en el ámbito científico-tecnológico de los/as académicos/as ecuatorianos/as retornados a partir de este Programa es incierta. En primer lugar, porque no contempla entre sus objetivos la repatriación sistemática de la población ecuatoriana altamente cualificada. En segundo lugar, porque frente a la actual crisis económica - debido al bajo precio del petróleo - y al recorte de recursos en todos los programas vinculados a la política de investigación, desarrollo e innovación $(I+D+I)$, se espera una reorientación de la política pública en general y cabría preguntarse si los profesionales ecuatorianos con proyectos de retorno estarán considerados como prioritarios dentro de estas reorientaciones.

Para concluir, en cuanto a las expectativas de futuro, un $21 \%$ de los/as encuestados/as pretende radicarse en Ecuador en un mediano y largo plazo, este perfil responde a investigadores/as entre 35 y 50 años que tienen una inserción muy precaria en sus lugares de origen, preferentemente en España y Venezuela. Un 25\% pretende mantener los vínculos entre Ecuador y la Europa Mediterránea como estrategias migratorias transnacionales, que se ven favorecidas por las ventajas que otorga el Gobierno de Ecuador en la regularización del estatus jurídico. Este hecho les permite organizar migraciones circulares dependiendo de las opciones laborales en los contextos de origen y de destino como una estrategia para enfrentar la crisis de la Universidad española e italiana actualmente.
Estos nuevos desplazamientos ponen en tela de juicio los discursos y prácticas de algunos gobiernos de la región sobre la llegada de "mano de obra cualificada" y la "circulación de talentos" que invisibilizan desigualdades entre estos/as trabajadores/as cualificados/as y sus pares en origen y entre otras categorías de migrantes. Asimismo, estas migraciones dan cuenta de estrategias migratorias individuales y familiares similares a aquellas seguidas por inmigrantes con una inserción laboral en sectores no cualificados y que no son reconocidas por las políticas que gestionan la "circulación de talentos", ni por los propios "migrantes cualificados". Por último, cabría reflexionar sobre las condiciones reales que presenta Ecuador en su mercado laboral para acoger estos proyectos migratorios con estrategias de asentamiento a mediano plazo y/o definitivas.

Nuestros hallazgos nos permiten afirmar que para profundizar en el análisis de la reconfiguración de los desplazamientos actuales en el sistema migratorio entre América Latina y Europa, es imprescindible utilizar la perspectiva de la interseccionalidad como herramientas teórico-metodológica potente que permite visibilizar la heterogeneidad y las desigualdades que se dan al interior de lo que se denominado "la migración cualificada".

\section{Notas}

1 Esta investigación se realizó durante la estancia en 2014 como Investigadora Prometeo de Claudia Pedone y de la estancia de investigación pre-doctoral de Yolanda Alfaro en la Escuela de Relaciones Internacionales "José Peralta" del Instituto de Altos Estudios Nacionales (IAEN), con la financiación del Programa Prometeo, SENESCYT, Gob. del Ecuador.

2 Sara Kalm (2008) en el capítulo quinto de su libro Governing Global Migration trata de repensar la política de migración a través de la conceptualización de la gubernamentalidad. Más específicamente, se ha propuesto utilizar el concepto de circulación para analizar la actual situación global de la migración y la política global de migración, con el fin de rastrear la racionalidad política de la gestión global de la migración.

3 Entendemos que la clase no se define por tanto por la suma de propiedades - sexo, edad, origen social o étnico, ingresos, nivel de instrucción - sino "por la estructura de las relaciones entre todas las propiedades pertinentes, que confiere su valor a cada una de ellas y a los efectos que ejerce sobre las prácticas" (Bourdieu, 1988: 112).

4 Para consultar en detalle las características y requisitos para acceder al Programa Prometeo ver: http://prometeo. educacionsuperior.gob.ec/.

5 Un estudio pionero en esta temática es la investigación de Yvonne Riaño (2012) donde plantea la falta de interés por la migración familiar y su impacto económico también ha eclipsado el estudio de las mujeres altamente cualificadas los proyectos migratorios familiares.

6 Para un detalle sobre las políticas de repatriación y/o atracción de extranjeros/as repatriados/as en Ecuador, 
Argentina, Brasil, Colombia y Chile, consultar Pedone (2014).

7 Es oportuno resaltar que la base de datos del Programa Prometeo otorga información sobre la nacionalidad, dónde se obtuvo el título y desde qué país se aplicó a la beca, por cual fue información privilegiada para elegir los sujetos de estudio para la muestra cualitativa.

\section{Referencias bibliográficas}

ANTHIAS, F. (2006), "Género, etnicidad, clase y migración: interseccionalidad y pertenencia translocalizacional", in P. Rodríguez (ed.), Feminismos periféricos, Granada, Editorial Alhulia, pp. 49-68.

BOURDIEU, P. (1988), La Distinción, Madrid, Taurus Alfaguara.

CHAPARRO F. e E. Arias (1970), La emigración de profesionales y técnicos colombianos y latinoamericanos 1960-1970, Colombia, Fondo colombiano de investigaciones científicas y proyectos especiales Francisco José de Caldas.

CRESSWELL, T. (2010), "Towards a Politics of Mobility", Environment and Planning D: Society and Space, 28, pp. 17-31.

DELGADO-WISE, R. (2014), "Migración Mexicana Altamente Calificada: Problemática y Desafíos", Debate. Observatorio Del Desarrollo, 2 (8), pp. 5-8.

ELLIOTT, A. e J. Urry (2010), Mobile Lives, New York, Routledge and Taylor \& Francis Group.

EZQUERRA, S. (2008), "Hacia un análisis interseccional de la regulación de las migraciones: la convergencia de género, raza y clase social", in E. Santamaría (ed.), Retos epistemológicos de las migraciones transnacionales, Barcelona, Anthropos.

FELDMAN-BIANCO, B., L. Sánchez et. al. (2011), "Introducción", in La Construcción del sujeto migrante en América Latina: Prácticas, representaciones y categorías, Quito, FLACSO/CLACSO/U, Alberto Hurtado, pp. 15-30.

KALM, S. (2008), "Governmentality", in Governing Global Migration, Lund Political Studies 153, Lund University, Department of Political Science, pp. 62-90.

KOOLHAAS M., V. Prieto e A. Pellegrino (2013), "Distribución territorial y características demográficas de la migración calificada, in Pellegrino, A.; J. Bengochea; M. Koolhaas (coord.), La migración calificada desde América Latina, Montevideo, Trilce, pp. 27-63.
MEZZADRA, S., N. de Genova e J. Pickles (eds.) (2015), "New Keywords: Migration and Borders", Cultural Studies, 29 (1), pp. 55-87.

MOHANTY, C. (2003), Feminism without Borders, Durham, Duke University Press.

OTEIZA, E. (1996), "Brain Drain: An historical and conceptual framework", International Scientific Migrations, Revista Redes, Universidad de Quilmes, 3 (7), pp. 101-20.

PEDONE, C. (2014), Migración cualificada y políticas públicas en América del Sur. El Programa Prometeo como estudio de caso, Quito, IAEN-SENESCYT. (Inédito)

PELLEGRINO, A. (2003), "Éxodo, movilidad y circulación: nuevas modalidades de la migración calificada", Estudios Sobre Migraciones Internacionales, Ginebra, Programa de migraciones internacionales, Oficina Internacional del Trabajo.

PNDBV 2013-2017 [Plan Nacional de Desarrollo del Buen Vivir 2013-2017], Quito, SENPLADES. Recuperado de: http://www.buenvivir.gob.ec/RODRÍGUEZ, A. (1986) "Los científicos sociales latinoamericanos como nuevo grupo de intelectuales", El Trimestre Económico, Fondo de Cultura Económica, 198, Abril-Junio., pp. 939-962, Disponible: http:// aleph.academica.mx/jspui/handle/56789/6145.

PROGRAMA PROMETEO (2014), Base de datos del Programa, Quito, SENESCYT. (documento inédito).

RIAÑO, Y. (2012), "The Invisibility of Family in Studies of Skilled Migration and Brain Drain", Diversities, 14, (1). Disponible en: www.unesco.org/shs/ diversities/vol14/issue1/art3

SECRETARIA NACIONAL DE PLANIFICACIÓN Y DESARROLLO (2013), Plan Nacional del Buen Vivir, Quito, Gobierno del Ecuador.

SENPLADES (2012) [Secretaría Nacional de Planificación y Desarrollo], Transformación de la Matriz Productiva. Quito, Ecuador: SENPLADES, 2012.

SHELLER, M. (2011), "Mobility", Sociopedia, Isa, pp. 1-12. Disponible en: http://www.sagepub.net/isa/resources/pdf/Mobility.pdf. DOI: $10.1177 / 205684601163$

SOLIMANO, A. (2013), Migraciones, Capital y Circulación de Talentos en la Era Global, México, Fondo de Cultura Económica.

VEGA, C.; C. Gómez e A. Correa (2014), "Circularidad migratoria entre Ecuador y España. Transformación educativa y estrategias de movilidad". Ponencia en el Seminario Internacional "Migración, Marcos Normativos y Políticas Públicas, nov, Quito, Escuela de Relaciones Internacionales, IAEN.

Recebido a 01/10/2015. Aceite para publicação a 04/12/2015.

Claudia Pedone (claudiapedone@yahoo.es). Universidad de Buenos Aires, Facultad de Filosofía y Letras, Instituto Interdisciplinario de Estudios de Género - CONICET. Puan 480, 1408 Ciudad Autónoma de Buenos Aires, Argentina. Yolanda Alfaro (corredijolatortuga@gmail.com). Universidad Autónoma de Zacatecas, Unidad Académica de Estudios del Desarrollo. Av. Preparatoria S/N, Col. Hidráulica, Apartado postal 3-138, 98065 Zacatecas, Zac., México. 